\title{
Secular Trends of Age at Menarche from 1985 to 2010 among Chinese Urban and Rural Girls
}

\author{
Hong Zhu ${ }^{1,2,3}$, Hong-Peng Sun ${ }^{1,2}$, Chen-Wei Pan ${ }^{1,2}$, Yong Xu ${ }^{1,2, *}$ \\ ${ }^{1}$ Department of Child and Adolescent Health, School of Public Health, Medical College of Soochow University, China \\ ${ }^{2}$ Center for Genetic Epidemiology \& Genomics, School of Public Health, Soochow University, China \\ ${ }^{3}$ Jiangsu Key Laboratory of Preventive and Translational Medicine for Geriatric Diseases, Soochow University, China
}

Copyright $(\mathcal{C} 2016$ by authors, all rights reserved. Authors agree that this article remains permanently open access under the terms of the Creative Commons Attribution License 4.0 International License

\begin{abstract}
We sought to determine whether average age at menarche (AAM) declined in Chinese girls during the past decades, and whether there were any differences of AAM among urban and rural girls in the mainland of China. The analysis of the study was based on Han schoolgirls aged 9 to 18 years in 22-30 provinces during 6 cycles $(1985,1991$, 1995, 2000, 2005 and 2010) of Chinese National Survey on Students' Constitution and Health. The average AAM overall was determined using probit analysis and compared between urban and rural areas. Correlation analyses and line regression models were used to explore the relationship of socioeconomic factors with AAM. In 1985-2010 the median AAM decreased rapidly at the speed of 3.9 months/decade for urban girls from 13.17 year $(95 \%$ CI, 13.14-13.20) to 12.35 year (95\% CI,11.65-13.01) and 5.95 months/decade for rural girls from 13.83 year $(95 \%$ CI, $13.80-13.85)$ to 12.59 years $(95 \% \quad \mathrm{CI}, 12.36-12.82)$. The urban-rural difference reduced continuously from 0.66 year in 1985 to 0.24 year in 2010. The average AAM in southeastern areas was lower than that in the northwest, both in urban girls and rural girls. Higher relative Gross Domestic Product per capita and residents' consumption level was consistently associated with increased likelihood of having reached menarche; the strength of associations for rural girls was stronger than that for urban girls. Our findings suggested presence of an obvious downward trend of AAM in Chinese girls from 1985 to 2010, both in urban and rural areas. The urban and rural distribution, regional difference and socioeconomic factors should be considered when interpreting trends in age at menarche in China.
\end{abstract}

Keywords Age at Menarche, Chinese Schoolgirls, Socio-economic Factors

\section{Introduction}

The first menses is a recognized milestone for female sexual maturity and the age at menarche (AAM) is the most widely used indicator. Information on AAM is essential for planning health promotion programs on women's health, especially in situations that early menarche is among the few established risk factors for breast cancer [1] and has possible relationships with overweight [2], cardiovascular disease [3], or sexual abuse [4]. Numerous evidences showed that AAM is likely to change with the development of society $[5,6]$, the degree and speed of which are not necessarily the same among different races, countries and regions. In the past 160 years, a systematic decrease in AAM was observed worldwide [7-9]. In recent decades, the age at onset of menarche and its variability have decreased $[10,11]$ or stabilized in some European countries [12, 13], but the downtrend of AAM is still ongoing in Africa [14, 15] and Asia [16]. In China, little is known so far about the secular trend and the urban-rural disparities of menarcheal age on a nationwide scale. Most existing studies on AAM of Chinese girls or women [17-19] had some limitations given the relative small sample size, limited regional composition, or short time spans they involved.

Socioeconomic status is assumed to be responsible for most if not all the decline in AAM by improving health, hygiene, nutrition, housing and employment $[9,20]$. With the largest population and a vast territory, China has had a booming economy over the past few decades especially since the 1980s. However, the influence of dramatic socioeconomic changes to AAM of Chinese girls has not been fully investigated. Considering huge urban-rural and regional disparities are distinctive features of its economic development, we speculate that there are some differences of AAM between urban and rural girls in China. But the questions as to what changes of urban-rural differences with time and whether or not the socioeconomic factors play different roles on AAM in urban girls and in rural girls remain to be determined.

In this study, we aimed to (1) estimate the secular trend of menarcheal age among Chinese girls from 1985 to 2010, (2) describe and compare the differences of AAM between 
urban and rural areas at the provincial and nationwide level, (3) determine any possible relationship of AAM and socioeconomic factors.

\section{Methods}

\subsection{Study Design and Sampling}

This study was conducted within the framework of Chinese National Survey on Students' Constitution and Health $(\mathrm{CNSSCH})$, a government's welfare project with a five-year interval since 1985. The serial studies of CNSSCH (1985, 1991, 1995, 2000, 2005 and 2010) followed a multistage, stratified, cross-sectional design to obtain nationally representative samples of the school-aged population, with the aim of assessing the development level and burdens of common health problems of children and adolescents in mainland China. The sampling procedure of $\mathrm{CNSSCH}$ has been described elsewhere previously [21-25]. In brief, it was a 3 -stage clustering process: (1) at the provincial level, the study participants were classified to four groups by gender (schoolboy vs. schoolgirl) and by region (urban vs. rural). The socioeconomic status (classified as "upper", "middle" and "low" at the regional level) of subjects were balanced in each group, (2) several primary and secondary school were randomly selected from 3 urban and 3 rural residential areas that were also randomly selected in each province, (3) students from 2 to 3 classes as clusters were selected at random in each grade of the selected schools. In the present study, we used the data of AAM investigations in Han girls aged 9 to 18. A total of $67349,29245,52306$, 45 186, 48 428, 42789 urban girls and 64 162, 29 194, 51 424, 44 901, 47 796, 42727 rural girls were selected in 1985, 1991, 1995, 2000, 2005 and 2010, respectively. It should be note that, for some reasons the survey conducted in 1991 was sampling from the higher socioeconomic classes, but the data was sufficient for the analyses. Our study was approved by the ethics committee of the Medical College of Soochow University in Suzhou, Jiangsu, China.

\subsection{Measurement of Age at Menarche}

Individual information of menarche was reported by the status quo method. The menstrual status data were collected by trained investigators (female physicians or school nurses) who would ask every schoolgirl aged $\geq 9$ the age of first menarche following a standard protocol. If an age was given, it was coded as a "yes" response and if the response was "haven't started yet," it was coded as a "no" response. These "yes-no" data were used to calculate AAM in the probit regression analysis. Probit regression analysis is commonly used to estimate AAM in a population whose menarche is emerging $[7,9]$ and this method is consistently used in six cycles of CNSSCH.

\subsection{Statistical Analysis}

Probit model was used to fit the proportion of girls of each age group (e.g., 9.00-9.99 years, 10.00-10.99 years) who had reached menarche. The population median AAM was the corresponding age at which $50 \%$ of girls in the population could be predicted to have reached menarche. The $95 \%$ confidence intervals (CI) of AAM for urban and rural groups were also estimated. The time trend of AAM between urban and rural girls in 1985-2010 was descriptively compared. Considering the subsequent surveys were sampling from additional provinces/ municipalities on the basis of study in 1985, we selected the AAM data from the same 22 provinces/ municipalities in 1985 and in 2010 to estimate the regional distribution of AAM by map labeling. Correlation analyses and simple linear regression analyses were used to determine the quantity relations of socioeconomic factors with AAM, taking the median AAM, GDP per capita and consumption level of every province/ municipality as a statistical unit, respectively. A 2 -sided $\mathrm{P}$ value $<0.05$ was considered significant. The data of real GDP per capita and the level of residents' consumption for 22 provinces/ municipalities corresponding to $\mathrm{CNSSCH}$ in 1985 and in 2010 was taken from the Chinese Bureau of National Statistics (NBS) and Statistical Yearbook of China[26]. Data analysis was conducted using SAS (Version 9.1; SAS Institute Inc., Cary, NC.USA) statistical software.

\section{Results}

The estimated medians of AAM in urban girls were 13.17 (95\% CI, 13.14-13.20) year, 13.01 (95\% CI, 12.94-13.07) year, 13.08 (95\% CI, 13.04-13.12) year, 12.74 (95\% CI, 12.06-13.36) year, 12.64 (95\% CI, 11.96-13.27) year, 12.35 (95\% CI, 11.65-13.01) year, in 1985, 1991, 1995, 2000, 2005 and 2010, respectively. Correspondingly, the medians of AAM in rural girls were 13.83 (95\% CI, 13.80-13.85) year, $13.63(95 \%$ CI, 13.57-13.69) year, $13.43 \quad(95 \%$ CI, 13.39-13.47) year, 13.14 (95\% CI, 12.66-13.57) year, 12.73 (95\% CI, 12.21-13.23) year and 12.59 (95\% CI, 12.36-12.82) year. The whole tendency of AAM in Chinese schoolgirls from 1985 to 2010 was decreasing (Figure 1). The overall acceleration rates of AAM during 25 years were 3.9 months/decade for urban girls and 5.95 months/decade for rural girls. The medians of AAM in rural girls were higher than those in urban girls across every survey year. The urban-rural differences of AAM reduced continuously from 0.66 year in 1985 to 0.24 year in 2010 and the minimum difference ( 0.09 year) appeared in 2005. 


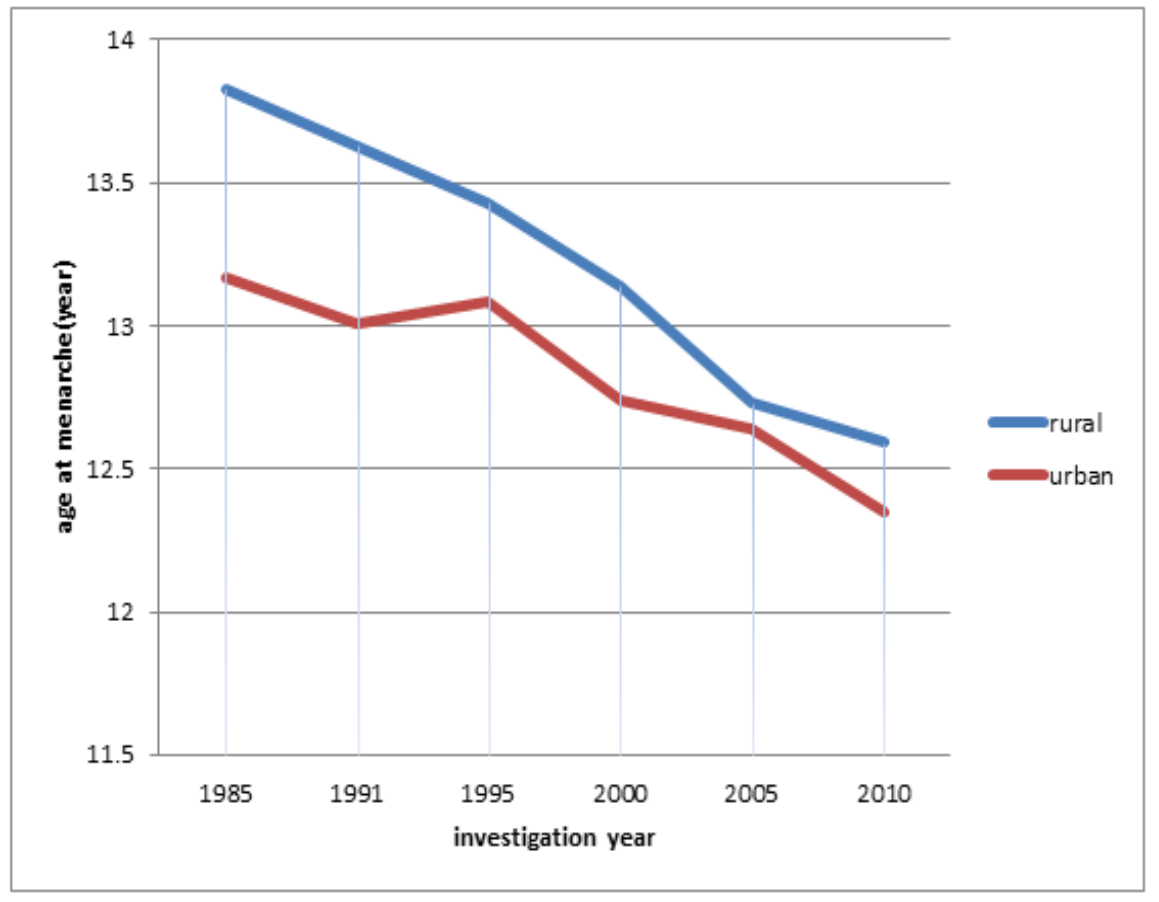

Figure 1. Trends in age at menarche in Chinese girls in 1985-2010

The differences of AAM between the highest and the lowest provinces were 0.9 year (Qinghai 13.51 year, Guangdong 12.61 year) for urban girls and 1.46 year (Qinghai 14.43 year, Beijing 12.97 year) for rural girls in 1985, which changed to 1.34 year (Qinghai 13.18 year, Liaoning 11.84 year) and 1.15 year (Ningxia 13.22 year, Heilongjiang 12.07 year) in 2010, respectively. Figure 2 shows the urban-rural differences of AAM that were generally smaller in 2010 than in 1985 across 22 provinces/municipalities. Several provinces showed a dramatic reduction and even a reversion of urban-rural differences of AAM like Qinghai, Heilongjiang and Zhejiang. Meanwhile, a 0.5-year gap of AAM was remained between urban and rural girls in Liaoning, Hebei, Ningxia and Shanxi both in 1985 and 2010.

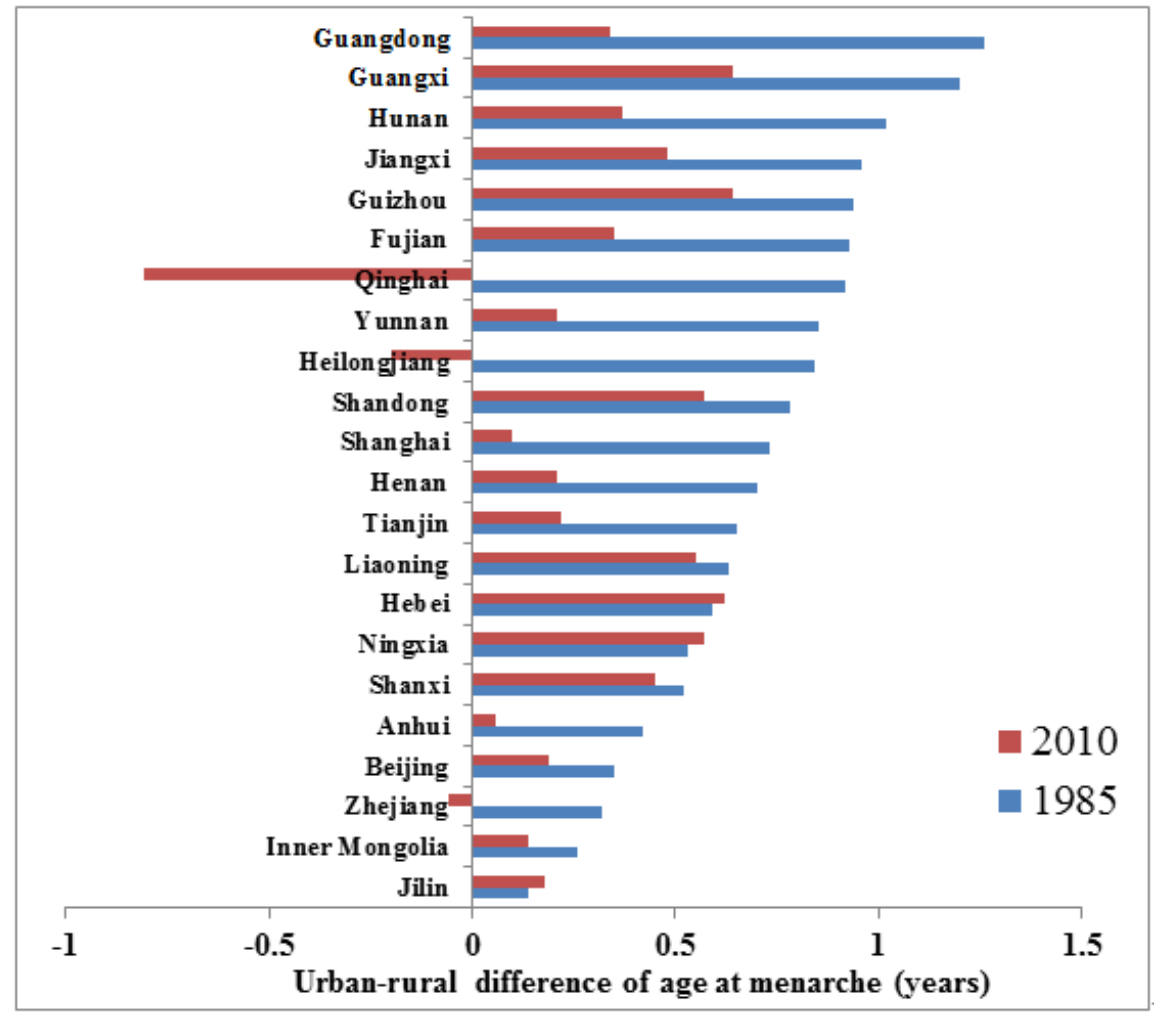

Figure 2. Urban-rural differences of age at menarche in 22 provinces/municipalities in 1985 and 2010 
Figure 3 presents the geographic distributions of median AAM in urban and rural areas across 22 provinces/municipalities from 1985 to 2010. Both in urban girls and rural girls, the average AAM in southeastern areas was lower than that in northwest. The hierarchy distribution of AAM for urban girls in 2010 was the most obvious.
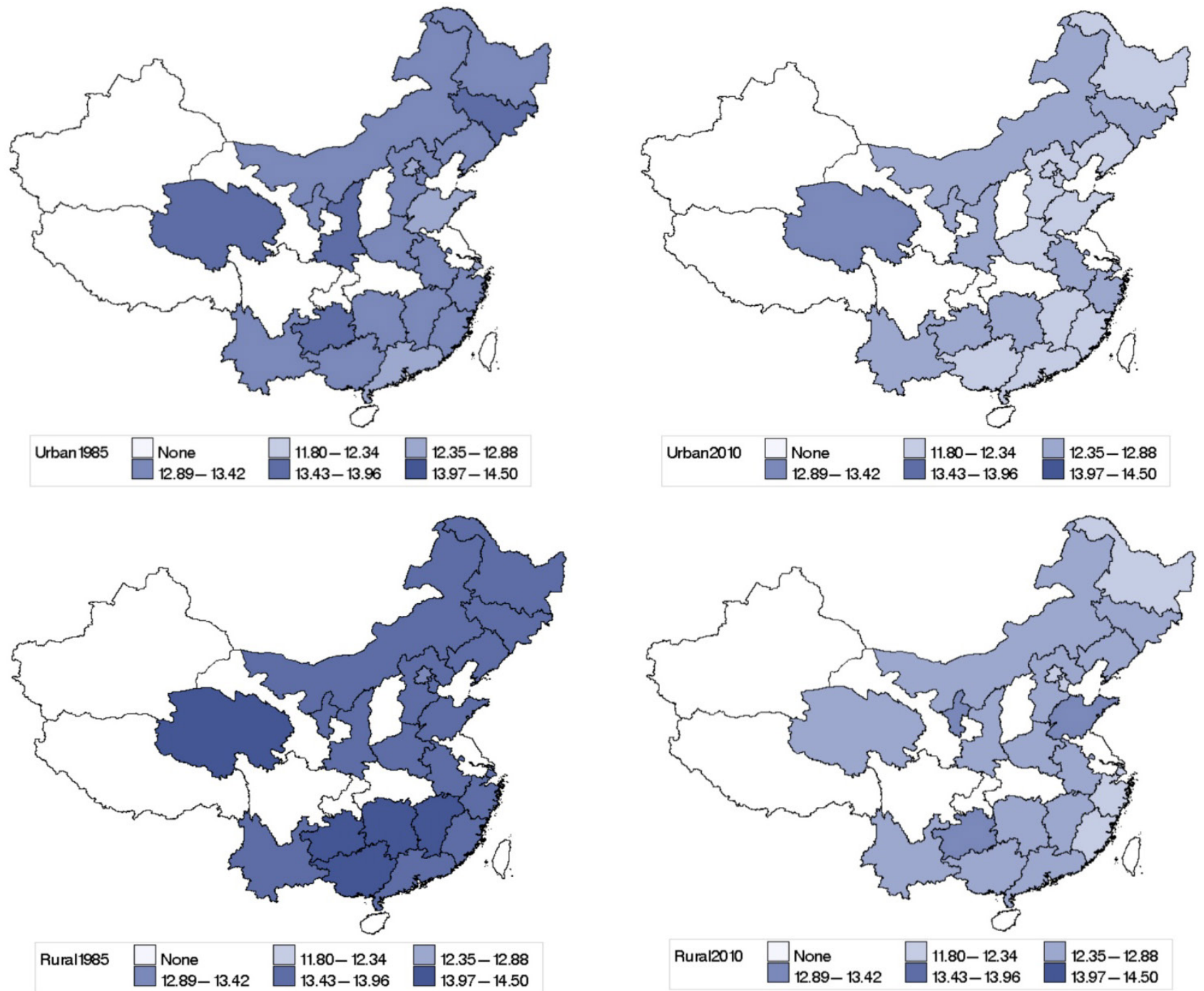

Figure 3. the geographic distribution of AAM

Correlation analyses showed that AAM was significantly and negatively associated with per capita GDP and consumption level, both in 1985 and 2010 (Table 1). The strength of associations between menarcheal age and per capita GDP or consumption level for rural girls was higher than that for urban girls (as Pearson's correlation coefficients revealed). According to the interrelated property we had reached several relevant linear regression equations (Figure 4). The coefficient of determination $\left(\mathrm{R}^{2}\right)$ for consumption level to AAM in the regression models was biggest in 2010 for rural girls $\left(\mathrm{R}^{2}=0.347\right.$, $\mathrm{P}=0.004)$.

Table 1. The Pearson's correlation coefficients of GDP per capita, consumption levels and median AAM of 22 Provinces/municipalities in 1985 and 2010.

\begin{tabular}{|c|c|c|c|c|c|c|c|}
\hline \multirow{4}{*}{$\begin{array}{c}\text { Age at } \\
\text { menarche }\end{array}$} & & \multicolumn{2}{|c|}{ GDP per capita } & \multicolumn{4}{|c|}{ Consumption levels } \\
\hline & & 1985 & 2010 & Urban 1985 & Rural 1985 & Urban 2010 & Rural 2010 \\
\hline & 1985 urban & $-0.49(\mathrm{p}=0.0197)$ & & $-0.41(p=0.0548)$ & & & \\
\hline & 1985 rural & $-0.58(p=0.0049)$ & & & $-0.59(p=0.004)$ & & \\
\hline & 2010 urban & & $-0.41(p=0.0576)$ & & & $-0.45(\mathrm{p}=0.0373)$ & \\
\hline & 2010 rural & & $-0.55(p=0.0074)$ & & & & $-0.58(p=0.0045)$ \\
\hline
\end{tabular}



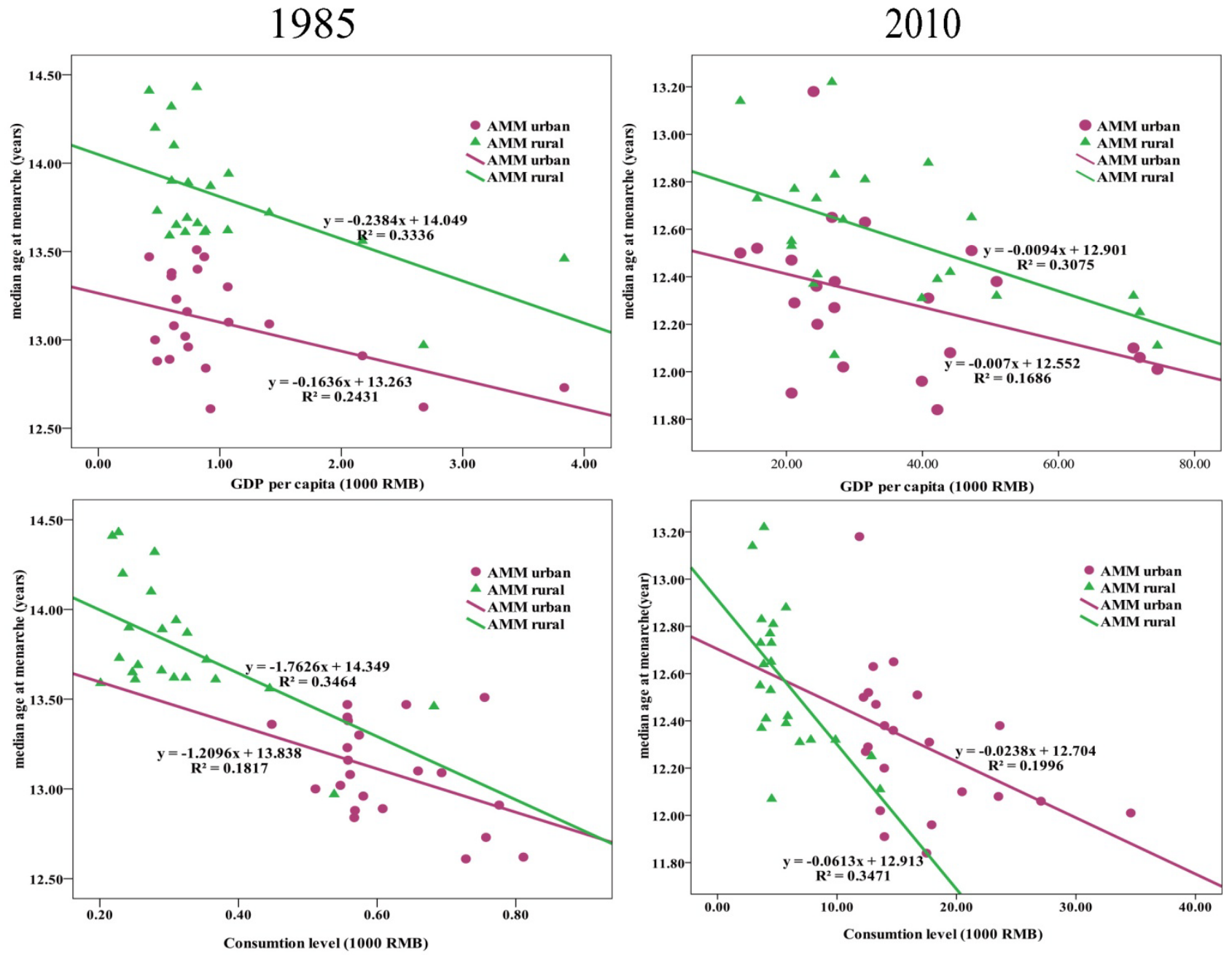

Figure 4. linear regression chart of AAM

\section{Discussion}

To our knowledge, the present study is among the first to estimate the secular trend of AAM in Chinese urban and rural girls and explore its possible associations with changes of socioeconomic circumstance.

The estimated median AAM in Chinese urban girls continuously declined from 13.17 to 12.35 year in 1985-2010, for rural girls it dropped from 13.83 to 12.59 year. The results were consistent with one similar but smaller study previously conducted in Beijing [18]. In that study, the median AAM of schoolgirls was 12.1 year and the accelerating speed in 1980-2004 was 4.2 and 9.6 months per decade for urban girls and rural girls, respectively. Our study's median AAM in 2010 is comparable with the age reported at the same period in Italy (12.4) [27], and South Korea (12.34) [28] but much lower than that in France (12.8) [5], Gambian ( 14.9) [6] and Turkey (13.3) [29]. One point of view is that the age at onset of menarche and its variability will stabilize at $13.0+/-0.5$ years $[12,13]$, but a report from UK showed that menarcheal age decreased again after a period of stabilization [30]. Meanwhile, although many countries have seen a fall in AAM in recent years, a very few has the drop been as fast as in China. For instance, reports from south Thailand [31], Israel [5], and the Netherlands [9] showed the AAM declined at the speed of 2 to 3 months/decade. We found the falling speed of AAM had slowed down since 2005; there was not a distinct plateau of AAM in Chinese schoolgirls however. Thus the conclusion cannot be made to date that the acceleration of AAM in Chinese girls will slow down or even stop in the near future.

Our findings also revealed some characteristics on AAM of Chinese girls, such as persistently existed but gradually reduced urban-rural differences, distinct provincial differences and a hierarchy reduction from northwest to southeastern national wide. As the difference of socioeconomic circumstance was the most apparent distinction between urban areas and rural areas, the actual faster speed of rural economic development during recent decades, the unbalanced economic growth both inside and outside the provinces, and the southeast costal region and municipalities were more developed than northwest areas in China, these characteristics on AAM accorded with the situations of modern Chinese economic development [32]. Our results attested that AAM was significantly and negatively associated with the level of socioeconomic 
development, and, the increasing of economic growth as GDP per capita and residents' consumption level reflected could at least partly explain the acceleration of AAM in Chinese girls. Interestingly, it seemed a stronger role of socioeconomic factors to AAM is located in rural areas in China. It can be speculated that the relatively more rapid improvement of health, hygiene, nutrition, housing and education in rural areas might attribute to these urban-rural variations.

Studies from countries with longer-standing economic prosperity have reported that early menarche is associated with higher risk of breast cancer [1], higher risk of death from all causes [33] and diabetes [34] in Caucasian women. The concrete quantitative evidence was similarly from China nowadays $[35,36]$ and the inverse associations between AAM and obesity or overweight were found as well [37, 38]. Thus in China, the long-term economic development of which is still in the initial stage, a constant attention to secular trend of menarcheal age, especially in rural areas, is required. More studies are warranted to further determine the mechanisms of secular trend of menarcheal age and its influences to reproductive characteristics, chronic diseases, and psychological behaviors in Chinese girls.

\section{Strengths and Limitations}

A major strength is the large study population, a 25-year time span and a national representative sample involved in our study. The CNSSCH is the largest series of growth surveillance in 6-22 year old adolescents in Chinese so far. By repeating the surveillance in 25 years with a 5 -year interval, secular trend of AAM can be explored clearly.

Due to the inevitable restrictions in research, this study has some limitations. Firstly, the actual menarche dates of the girls were not used to measure the mean of AAM. This might cause information bias to some extent. However, the "status quo" method in a large study population whose menarche is emerging is considered to be even more applicable and reliable than the recall method. Secondly, the cross-sectional nature of the survey and the utilization of macro socioeconomic data make it impossible to establish a causal relationship between urban/rural residence and socioeconomic factors. Thirdly, China is not a single ethnic nation but a 56-ethnic country. The secular trend of AAM in Han girls might not completely represent the national situation, even if the Han ethnicity account for more than $90 \%$ of the total populations. Forth, although the estimated medians of AAM in each cycles of $\mathrm{CNSSCH}$ were standardized according to the age distribution of 1985 population for the purpose of comparison, it is possible that unintentional errors occurred when comparing the secular trend and the urban-rural differences of AAM.

\section{Conclusions}

In summary, the median of menarcheal age in Chinese girls is decreasing from 1985 to 2010, both in urban and rural areas. There are obvious urban-rural differences of AAM in Chinese girls and they were with a tendency to narrow both on the nationwide and provincial scale. Our study also suggested that menarcheal age was associated with changes of socioeconomic circumstance in Chinese girls. These data are essential to guide future health care and clinical management in China, and provide important public health implications for other developing countries with rapid economic growth.

\section{Acknowledgments}

This work is partly supported by National Nature Science Foundation of China (No.81072312) to Yong Xu.

\section{Author Contributions}

Conceived and designed the study: YX

Data collecting: HZ

Analyzed the data: HPS, HZ, CWP

Wrote the paper: HZ

All the authors were involved in organizing and refining the article.

Conflict of Interest: none declared

\section{REFERENCES}

[1] Ritte R, Tikk K, Lukanova A, et al. Reproductive factors and risk of hormone receptor positive and negative breast cancer: a cohort study. BMC cancer. 2013;13:584.

[2] Castilho SD, Nucci LB. Age at menarche in schoolgirls with and without excess weight. Jornal de pediatria. 2015;91:75-80.

[3] Mueller NT, Odegaard AO, Gross MD, et al. Age at menarche and cardiovascular disease mortality in Singaporean Chinese women: the Singapore Chinese Health Study. Annals of epidemiology. 2012;22:717-22.

[4] Boynton-Jarrett R, Wright RJ, Putnam FW, et al. Childhood abuse and age at menarche. The Journal of adolescent health : official publication of the Society for Adolescent Medicine. 2013;52:241-7.

[5] Flash-Luzzatti S, Weil C, Shalev V, et al. Long-Term Secular Trends in the Age at Menarche in Israel: A Systematic Literature Review and Pooled Analysis. Hormone research in paediatrics. 2014;81:266-71.

[6] Vecek N, Vecek A, Zajc Petranovic M, et al. Secular trend of menarche in Zagreb (Croatia) adolescents. European journal of obstetrics, gynecology, and reproductive biology. 2012;160:51-4.

[7] Anderson SE, Must A. Interpreting the continued decline in 
the average age at menarche: results from two nationally representative surveys of U.S. girls studied 10 years apart. The Journal of pediatrics. 2005;147:753-60.

[8] Woronkowicz A, Cichocka BA, Kowal M, et al. Physical development of girls from Krakow in the aspect of socioeconomical changes in Poland (1938-2010). American journal of human biology : the official journal of the Human Biology Council. 2012;24:626-32.

[9] Talma H, Schonbeck Y, van Dommelen P, et al. Trends in menarcheal age between 1955 and 2009 in the Netherlands. PloS one. 2013;8:e60056.

[10] Atay Z, Turan S, Guran T, et al. Puberty and influencing factors in schoolgirls living in Istanbul: end of the secular trend? Pediatrics. 2011;128:e40-5.

[11] Cabrera SM, Bright GM, Frane JW, et al. Age of thelarche and menarche in contemporary US females: a cross-sectional analysis. Journal of Pediatric Endocrinology and Metabolism. 2014;27:47-51.

[12] Gaudineau A, Ehlinger V, Vayssiere C, et al. [Age at onset of menarche: Results from the French Health Behaviour in School-aged Children study]. Gynecologie, obstetrique \& fertilite. 2010;38:385-7.

[13] Lalys L, Pineau JC. Age at menarche in a French population of young schoolgirls. Pediatrics International. 2014;56:601-4.

[14] Prentice S, Fulford AJ, Jarjou LM, et al. Evidence for a downward secular trend in age of menarche in a rural Gambian population. Annals of human biology. 2010;37:717-21.

[15] Aryeetey R, Ashinyo A, Adjuik M. Age of menarche among basic level school girls in Medina, Accra. African journal of reproductive health. 2011;15:103-10.

[16] Cho GJ, Park HT, Shin JH, et al. Age at menarche in a Korean population: secular trends and influencing factors. European journal of pediatrics. 2010;169:89-94.

[17] Sun Y, Tao FB, Su PY, et al. National estimates of the pubertal milestones among urban and rural Chinese girls. Journal of Adolescent Health. 2012;51:279-84.

[18] Chen FF, Wang YF, Mi J. Timing and secular trend of pubertal development in Beijing girls. World journal of pediatrics : WJP. 2014;10:74-9.

[19] Song Y, Ma J, Hu PJ, et al. [Geographic distribution and secular trend of menarche in 9-18 year-old Chinese Han girls]. Beijing da xue xue bao Yi xue ban = Journal of Peking University Health sciences. 2011;43:360-4.

[20] Kaplowitz PB. Link between body fat and the timing of puberty. Pediatrics. 2008;121 Suppl 3:S208-17.

[21] Ji CY, Chen TJ. Empirical changes in the prevalence of overweight and obesity among Chinese students from 1985 to 2010 and corresponding preventive strategies. Biomedical and Environmental Sciences. 2013;26:1-12.

[22] Ji CY, Chen TJ, Sun X. Secular changes on the distribution of body mass index among Chinese children and adolescents, 1985-2010. Biomedical and Environmental Sciences. 2013;26:520-30
[23] Song Y, Wang HJ, Ma J, et al. Secular trends of obesity prevalence in urban Chinese children from 1985 to 2010: gender disparity. PloS one. 2013;8:e53069.

[24] Chen TJ, Ji CY. Secular change in stature of urban Chinese children and adolescents, 1985-2010. Biomedical and Environmental Sciences. 2013;26:13-22.

[25] Sun H, Ma Y, Han D, et al. Prevalence and trends in obesity among China's children and adolescents, 1985-2010. PloS one. 2014;9:e105469.

[26] Chinese Bureau of National Statistics (NBS) and Statistical Yearbook of China, URL: http://www.stats.gov.cn/

[27] Rigon F, Bianchin L, Bernasconi S, et al. Update on age at menarche in Italy: toward the leveling off of the secular trend. Journal of Adolescent Health. 2010;46:238-44.

[28] Fujii K, Tanaka N. Critical period for menarche derived by the wavelet interpolation method from changes in BMI with age in South Korean girls. Journal of human ergology. 2010;39:89-97.

[29] Adali T, Koc I. Menarcheal age in Turkey: secular trend and socio-demographic correlates. Annals of human biology. 2011;38:345-53.

[30] Morris DH, Jones ME, Schoemaker MJ, et al. Secular trends in age at menarche in women in the UK born 1908-93: results from the Breakthrough Generations Study. Paediatric and perinatal epidemiology. 2011;25:394-400.

[31] Jaruratanasirikul S, Sriplung H. Secular trends of growth and pubertal maturation of school children in Southern Thailand. Annals of human biology. 2014:1-8.

[32] Xie Y, Zhou X. Income inequality in today's China. Proceedings of the National Academy of Sciences of the United States of America. 2014;111:6928-33

[33] Charalampopoulos D, McLoughlin A, Elks CE, et al. Age at menarche and risks of all-cause and cardiovascular death: a systematic review and meta-analysis. American journal of epidemiology. 2014;180:29-40.

[34] Elks CE, Ong KK, Scott RA, et al. Age at menarche and type 2 diabetes risk: the EPIC-InterAct study. Diabetes care. 2013;36:3526-34

[35] Qiu C, Chen H, Wen J, et al. Associations between age at menarche and menopause with cardiovascular disease, diabetes, and osteoporosis in Chinese women. Journal of Clinical Endocrinology and Metabolism. 2013;98:1612-21.

[36] Conway BN, Shu XO, Zhang X, et al. Age at menarche, the leg length to sitting height ratio, and risk of diabetes in middle-aged and elderly Chinese men and women. PloS one. 2012; $7: \mathrm{e} 30625$

[37] Al-Awadhi N, Al-Kandari N, Al-Hasan T, et al. Age at menarche and its relationship to body mass index among adolescent girls in Kuwait. BMC public health. 2013;13:29.

[38] Guo X, Ji C. Earlier menarche can be an indicator of more body fat: study of sexual development and waist circumference in Chinese girls. Biomedical and Environmental Sciences. 2011;24:451-8. 